\title{
Anomalous Electrokinetics on Hydrophobic Surfaces: Effects of Ion Specificity and Interfacial Water Structure
}

\author{
Qianqian $\mathrm{Cao}^{1,2, *}$ and Roland R. Netz ${ }^{2, *}$ \\ ${ }^{1}$ College of Mechanical and Electrical Engineering, Jiaxing University, Jiaxing \\ 314001, P.R. China \\ ${ }^{2}$ Fachbereich Physik, Freie Universität Berlin, 14195 Berlin, Germany \\ *Corresponding authors. E-mail: qqcao@mail.zjxu.edu.cn (QC); \\ rnetz@physik.fu-berlin.de (RN)
}

\begin{abstract}
We study the electrokinetic transport behavior of water molecules and ions in hydrophobic graphene nanochannels with variable surface charge densities and the interfacial water structure based on detailed molecular dynamics simulations. The interfacial water structure, described by the water density, hydrogen bonding, diffusion, distribution of the $\mathrm{OH}$ bond and dipole orientations, are strikingly influenced by the surface charge. We find anomalous electrostatic effects which depend on the distribution of counterions close to the surface, ion-specific effects and interfacial water structure. On a negatively charged surface, the attraction of $\mathrm{Na}^{+}$ions towards the graphene layer enhances the interfacial friction. In contrast, if the surface is positively charged, high surface charge density triggers an anomalous enhancement of electroosmotic flow, accompanied by an abrupt change of the interfacial water structure. At high surface charge densities, the mobility of the interfacial water at the positively charged surfaces is suppressed more strongly compared to the negatively charged surface. Our results imply new physics in electrokinetic transport when both negatively and positively charged surfaces are considered.
\end{abstract}




\section{Introduction}

Surface properties are of fundamental importance in controlling the molecular transport in nanofluidic systems by mediating the solvent structure at solid/liquid interfaces [1-4]. In hydrophobic nanochannels, the flow resistance due to interactions between fluid molecules and the substrate is significantly weakened compared to hydrophilic surfaces. As a consequence, the flow rate of water through carbon nanotubes is dramatically enhanced $[5,6]$. The interfacial depletion region of water molecules close to the hydrophobic surface is at the origin of new transport phenomena that are different from hydrophilic channels. Many studies demonstrated that the transport dynamics of water along hydrophobic surfaces depends on the structure of water molecules in the depletion region [7]. Computer simulations revealed that dramatic enhancement of the flow rate of water in carbon nanotubes is caused by the water orientation and interfacial hydrogen bonding [8]. Further works confirmed that the curvature of carbon nanotube membranes also strongly influences the interfacial friction of water $[9,10]$.

The fluidic transport in nanochannels can be driven by various mechanisms. Typical driving forces are pressure, electric fields, surface tension gradients, osmotic pressure, temperature and concentration gradients $[11,12]$. Electroosmotic flow can be generated when the ions within the electrical double layer (EDL) near a charged surface are driven by an external electric field. Compared to other driving modes, the electroosmotic transport provides a rapid and efficient mean for modulating the flow in extremely confined environments. The EDL is formed due to attractive electrostatic interaction between counterions and the charged surface. The static and dynamic properties of charged surface systems can be related to the structure of the EDL and the interactions between molecules in the EDL and the substrate [13-15]. Furthermore, understanding the electrohydrodynamics in the EDL is critical for numerous applications, such as separation [16,17], fuel cell performance [18], energy storage and conversion [19-21], contaminant removal [22], and manipulation of single molecules [23,24]. In addition to 
the surface properties, the EDL structure also depends on salt concentration and ion types. Typically, the interaction of different ions with the surface and specific ionic hydration effects strongly influence the EDL structure and as a consequence change the transport behavior of solvent molecules and ions [25-28].

Electrokinetic transport in hydrophobic nanochannels has been investigated theoretically and computationally $[25,27,29-38]$. A thin vapor phase exists close to the surface and amplifies interfacial slippage by preventing the fluid from being directly exposed to the surface roughness. Chakraborty developed a generalized mesoscale model and predicted a universal dependence of the interfacial electromechanics regardless of the details of the bulk flow pattern [32]. Huang et al. identified ion-specific anomalous flow provoked by the strong attraction of large ions to a hydrophobic surface [25]. Computer simulations on the electroosmotic flow of $\mathrm{NaCl}$ solutions across charged carbon nanotubes indicated that the surface charge density has a significant effect on the flow characteristics depending on the adsorption behavior of different counterions [27]. A high-ionic-strength electroosmotic flow was also observed in uncharged nanochannels [33]. Through the formulation of the electrohydrodynamic slip boundary condition, the hydrodynamic properties of the EDL and the fluid flow were demonstrated to depend on both confinement and mobility of surface charges [38]. The zeta potential $\zeta$ is a critical parameter of the EDL. It represents the difference of the electrokinetic potential between the bulk solution and the shear plane for polar surfaces with zero slip. However, hydrophobic interactions are characterized by a finite surface slip. As a consequence, the zeta potential is amplified owing to the existence of slippage at the hydrophobic surfaces [29]. The dynamic origin for the amplification of the zeta potential was also demonstrated experimentally [39]. For strongly hydrophobic surfaces, the slip length becomes larger than the characteristic dimension of the nanochannel, and the hydrodynamics is mainly dependent on the molecular friction in the interfacial region, and not on the solvent viscosity [9].

In nanofluidic systems, classical continuum assumptions inherent in continuum hy- 
drodynamics and the mean-field Poisson-Boltzmann (PB) theory may not work well in describing the flow field and the ion distribution under specific conditions. For example, the classical PB formalism stemming from purely electrostatic interactions between different charged species offers only a poor description for strong surface charges and high ion valency [40]. Furthermore, the effects of significant fluctuations in the ion and water densities close to the surface and discrete effects that come from the finite molecular sizes, which are not considered in the original PB theory [41-43], become non-negligible in narrow fluidic environments. In addition, the flow reversal triggered by the charge inversion [44-46] as well as other anomalous transport phenomena caused by ion-specific effects $[25,28,47]$ may also not be captured by classical continuum theories. Recently, Rezaei et al. studied the electroosmotic flow of an $\mathrm{NaCl}$ solution along silicon surfaces with different surface charge densities through computer simulation [45]. It was found that the flow velocity first rises and then decreases until a flow reversal occurs as the surface charge density increases. The charge inversion is caused by the immobilization of adsorbed counterions and leads to the flow reversal [44]. In carbon nanotubes, the flow velocity is more than three orders of magnitude higher than predicted by continuum hydrodynamics models [6]. In narrower nanochannels, a distinct change of the viscosity in the channel also complicates standard continuum approaches. Though some corrections can be included into classic theories [37], there exist still deficiencies in the model assumptions or when applying them to charged surfaces with complex molecular details. Molecular dynamics (MD) accounts for molecular details which are important to investigate the fluid flow in nanosized channels or pores, such as the discontinuity of matter and atomic interactions.

In this work, through MD simulations, we analyze the anomalous electrohydrodynamic behavior in a charged graphene nanochannel and the water structure at the solid/liquid interface. Our study shows that for negatively charged surfaces, the water transport undergoes fast flow, strongly reduced flow and finally flow reversal as the surface charge density increases, similar to previous works $[44,48]$. However, when the 
surface is positively charged, an anomalous enhanced flow is identified at high surface charge densities, and an abrupt change in the interfacial water structure induces a transition in the zeta potential, which has not been reported in previous works. The variation of surface charge density also leads to some interesting results for the ion concentration and water structure in the interfacial region. Besides the electrokinetic transport in hydrophobic nanochannels (such as carbon nanotubes [27,49] and boron nitride nanotubes [21]), our results may be useful to further understand the electrokinetic properties on the surfaces of carbon-based material electrodes, which are widely used in energy storage systems. The EDL structures for mesoporous carbon electrodes [50] and flat graphite electrodes [51] have been previously studied through computer simulations. However, how the surface charge density influences the interfacial structures and dynamics remained unclear in such systems. The present study suggests that in charged hydrophobic nanochannels, new transport mechanisms still remain to be explored at the molecular level which depend on details of the liquid interfacial properties and the surface structure.

\section{Simulation Model and Method}

The electrolyte is confined between two frozen graphene walls, each of which includes two layers of graphene separated by a distance of $0.335 \mathrm{~nm}$. Only carbon atoms in the inner layer are charged. Each graphene sheet consists of 1008 carbon atoms. The dimensions of the simulation box parallel to the walls are $l_{x} \times l_{y}=5.105 \mathrm{~nm} \times 5.158 \mathrm{~nm}$. Periodic boundary conditions are applied along these two directions. The separation between the two innermost graphene layers along the $z$ direction is approximately $l_{z}=7.1 \mathrm{~nm}$. About 5900 water molecules are included in the simulation box. All carbon atoms in the inner layer are positively or negatively charged from 0.01 to 0.1 elementary charge per atom, corresponding to a range of the surface charge density of $\left|\sigma_{s}\right|=0.061 \mathrm{C} / \mathrm{m}^{2}$ to $0.613 \mathrm{C} / \mathrm{m}^{2}$. For the lowest surface charge density investigated, there is only a total charge of $10 e$ in each charged layer. 
Non-bonded interactions between molecules are described by a combination of LennardJones (LJ) and Coulombic potentials

$$
U_{\alpha \beta}(r)=4 \epsilon_{\alpha \beta}\left[\left(\frac{\sigma_{\alpha \beta}}{r}\right)^{12}-\left(\frac{\sigma_{\alpha \beta}}{r}\right)^{6}\right]+\frac{1}{4 \pi \varepsilon_{0}} \frac{q_{\alpha} q_{\beta}}{r}
$$

where $r$ is the distance between atoms $\alpha$ and $\beta$ with partial charge $q_{\alpha}$ and $q_{\beta}, \epsilon_{\alpha \beta}$ and $\sigma_{\alpha \beta}$ are the characteristic LJ energy and size parameters, respectively, $\varepsilon_{0}$ is the permittivity of vacuum. The shifted LJ interactions are truncated at $1.1 \mathrm{~nm}$. The carbon atoms have the same potential parameters as the carbon atoms in benzene obtained from the Amber99 force field [52]. The LJ parameters for $\mathrm{Na}^{+}$and $\mathrm{Cl}^{-}$ions are taken from Ref. [53]. The Lorentz-Berthelot mixing rule is used to calculate the potential parameters between dissimilar particles. The parameters for the $\mathrm{O}-\mathrm{C}$ pairs are chosen as $\epsilon_{o c}=0.105 \mathrm{kcal} / \mathrm{mol}$ and $\sigma_{o c}=0.319 \mathrm{~nm}$, based on the work of Werder et al. [54], which makes an accurate prediction for the contact angle of water on graphite surfaces. Water molecules are modeled by using the extended Simple Point Charge (SPC/E) model [55], and the hydrogen atoms have no LJ interaction. The SHAKE algorithm [56] is used to constrain the water geometry. The salt $(\mathrm{NaCl})$ concentration is fixed at $c_{0}=0.71 \mathrm{M}$. Extra $\mathrm{Na}^{+}$or $\mathrm{Cl}^{-}$counterions are added to neutralize the charged graphene layers. Long-ranged electrostatic interactions are treated by the particle-particle particle-mesh (PPPM) solver [57] with a real-space cutoff of $1.1 \mathrm{~nm}$ and a maximal error of $10^{-4}$ for the force calculation in reciprocal space. To compute the Coulomb interaction of the system with a finite length in the $z$ direction, an empty volume with the height of $3 l_{z}$ is inserted along the $z$ direction, and a correction term is added [58].

The Large-scale Atomic/Molecular Massively Parallel Simulator (LAMMPS) [59] is used to perform simulations in the NVT ensemble. The system is coupled to a NoséHoover thermostat $[60,61]$ to regulate the temperature of the fluid at $300 \mathrm{~K}$ with a time constant of $0.1 \mathrm{ps}$. An external electric field is applied along the $x$ direction to induce an electroosmotic flow. To avoid biasing the flow velocity profile, only the velocity 
components along the $y$ and $z$ directions are thermostated. The system is equilibrated for $1 \mathrm{~ns}$ in the absence of the electric field. A run of $8 \mathrm{~ns}$ under the electric field is performed to reach a steady flow state. After that, the system is simulated for $15 \mathrm{~ns}$. The equations of motion are integrated using the velocity-Verlet algorithm with a time step of 2 fs.

\section{Interfacial Electrical Potential}

We describe how to calculate the electrostatic potential in a planar double layer system with inhomogeneous dielectric properties. The Poisson equation in the presence of spatial variations of the dielectric profile $\varepsilon_{\perp}(z)$ is given by

$$
\frac{\mathrm{d}}{\mathrm{d} z}\left[\varepsilon_{\perp}(z) \frac{\mathrm{d}}{\mathrm{d} z} \psi(z)\right]=-\frac{\rho(z)}{\varepsilon_{0}}
$$

with the electrostatic potential $\psi(z)$ and the charge density $\rho(z)$. We model the spatially varying dielectric profile using a step function [37]

$$
\varepsilon_{\perp}(z)= \begin{cases}\varepsilon_{\text {int }} & \text { if } z_{w}<z<z_{\text {int }} \\ \varepsilon_{\text {bulk }} & \text { if } z>z_{\text {int }}\end{cases}
$$

where $\varepsilon_{\text {int }}$ and $\varepsilon_{\text {bulk }}$ are the interfacial and bulk water dielectric constants, respectively. $z_{w}$ is the position of the wall, and $z_{\text {int }}$ the position of the interfacial layer. We calculate the thermodynamically defined interface position by a Gibbs dividing surface construction. The Gibbs dividing surface is given by

$$
z_{G D S}=z_{w}+\int_{z_{w}}^{z_{b}} \frac{\rho\left(z_{b}\right)-\rho(z)}{\rho\left(z_{b}\right)-\rho\left(z_{w}\right)} \mathrm{d} z
$$

where $\rho(z)$ is the water density, and $z_{b}$ is the position of the bulk liquid. Here, we take $z_{i n t}=z_{G D S}$, and then obtain the width of the interfacial layer $\Delta z_{\text {int }}=z_{\text {int }}-z_{w}$. It was found that the electrostatic attraction between the negatively charged surface 
and the hydrogen atoms with positive partial charges leads to a smaller $\Delta z_{\text {int }}$ due to a reorientation of the water molecules at the interface. The boundary conditions on the charged surface $z=z_{w}$ and far away from the surface $z \rightarrow \infty$ are expressed as

$$
\begin{gathered}
\left.\frac{\mathrm{d} \psi(z)}{\mathrm{d} z}\right|_{z=z_{w}}=-\frac{\sigma_{0}}{\varepsilon_{i n t} \varepsilon_{0}} \\
\left.\psi(z)\right|_{z \rightarrow \infty}=0
\end{gathered}
$$

where $\sigma_{0}$ is the surface charge density. We use continuous boundary conditions of the electrostatic potential and the displacement field at $z_{\text {int }}$

$$
\begin{gathered}
\left.\psi(z)\right|_{z=z_{i n t}-0}=\left.\psi(z)\right|_{z=z_{i n t}+0} \\
\left.\varepsilon_{i n t} \frac{\mathrm{d} \psi(z)}{\mathrm{d} z}\right|_{z=z_{i n t}-0}=\left.\varepsilon_{b u l k} \frac{\mathrm{d} \psi(z)}{\mathrm{d} z}\right|_{z=z_{i n t}+0}
\end{gathered}
$$

To calculate the electrostatic potential, we first need to determine the charge density $\rho(z)$. On one hand, the charge distribution can be extracted from the MD data. On the other hand, based on the continuum description the concentrations of cations and anions obey the Boltzmann distribution. By solving the PB equation, the analytical expression of the interfacial potential $\Psi_{\text {int }}$ that is the electrostatic potential at $z=z_{\text {int }}$, satisfying the above boundary conditions is obtained [62]

$$
\left.\frac{\mathrm{d} \Psi}{\mathrm{d} z}\right|_{z=z_{w}}=\left\{\begin{array}{c}
-2 \Gamma \mathrm{ds}\left(\Gamma \Delta z_{\text {int }}-F\left(\arccos \left[-\tanh \left(\Psi_{\text {int }} / 2\right)\right] \mid 1-p\right) \mid 1-p\right) \\
2 \sqrt{p} \Gamma \mathrm{dc}\left(\sqrt{p} \Gamma \Delta z_{\text {int }}+F\left(\arcsin \left[-\tanh \left(\Psi_{\text {int }} / 2\right)\right] \mid 1-p^{-1}\right) \mid 1-p^{-1}\right)
\end{array}\right.
$$

where $\Psi=\psi e / k_{B} T$ is the dimensionless potential, $\Gamma=\kappa \sqrt{\varepsilon_{b u l k} / \varepsilon_{\text {int }} \mathrm{e}^{\alpha}}$ with the inverse Debye length $\kappa=e \sqrt{2 c_{0} / \varepsilon_{0} \varepsilon_{b u l k} k_{B} T}$ and the ion-surface interaction parameter $\alpha$, and the integration constant $p=\left(\mathrm{e}^{\alpha} \varepsilon_{\text {bulk }} / \varepsilon_{\text {int }}-1\right)\left(\cosh \Psi_{\text {int }}-1\right) / 2 \cdot \operatorname{ds}(u \mid m)$ and $\operatorname{dc}(u \mid m)$ are Jacobian elliptic functions, and $F(\varphi \mid m)$ is the incomplete elliptic integral of the first kind. In the calculations, we set the parameters to $\varepsilon_{i n t}=1, \varepsilon_{\text {bulk }}=78, T=298 \mathrm{~K}$, 
and $\alpha=0$. More details can be found in Ref. [62].

\section{Results and Discussion}

We first discuss the effect of the surface charge density $\sigma_{s}$ on the electroosmotic velocity. Figure 1a presents the average flow velocity $u_{e}$ as a function of $\sigma_{s}$ at $E=0.1 \mathrm{~V} / \mathrm{nm}$. $u_{e}$ is defined as the average velocity of water molecules not including the near-surface region $(<0.7 \mathrm{~nm}$ from the charged surface). For the positively charged (PC) surface, we observe an anomalous dependence of $u_{e}$ on $\sigma_{s}$ : the flow is enhanced suddenly when

$\sigma_{s}$ exceeds $0.46 \mathrm{C} / \mathrm{m}^{2}$ corresponding to a surface charge of 0.075 e per atom; further increasing $\sigma_{s}$ beyond $0.52 \mathrm{C} / \mathrm{m}^{2}$ leads to a decrease of $u_{e}$. The sudden increase of flow velocity corresponds to an increase of the slip length $b$. The zeta potential of a hydrophobic surface can be calculated by the formula $\zeta=V_{0}\left(1+\kappa_{\text {eff }} b\right)$ with the surface potential $V_{0}$ and the effective inverse Debye length $k_{\text {eff }}[29,63]$. Consequently, an increase of the slip length leads to a transition in the zeta potential, which is demonstrated to be induced by an abrupt change in the interfacial water structure. The mechanism of anomalous flow will be discussed further below. When the surface is negatively charged (NC), the velocity is suppressed significantly at high surface densities $\left|\sigma_{s}\right|>0.35 \mathrm{C} / \mathrm{m}^{2}$. In previous works of Qiao et. al [27], the bulk transport was found to be negligible for negatively charged single-wall carbon nanotubes with discrete surface charges already at a surface charge density $\left|\sigma_{s}\right|=0.076 \mathrm{C} / \mathrm{m}^{2}$. The difference is caused by the surface curvature and the discreteness of surface charges corresponding to a set of selected carbon atom carrying a unit charge in that work. Note that the flow velocity for the NC surface is much larger compared to that for the $\mathrm{PC}$ surface at relatively low surface charge density $\sigma_{s}=0.245 \mathrm{C} / \mathrm{m}^{2}$. The present study indicates that the transport behavior of the fluid along the charged graphene surface is significantly influenced by the density and distribution of surface charges. We also examine the liquid/solid friction coefficient $\lambda=F / u_{e} A$ with $A$ the contact area and $F$ the total electrostatic force exerted on cations and anions, shown by open 
symbols in Figure 1. In the strong slip limit, the interfacial friction and not the solvent viscosity controls the fluid dynamics. In particular, the velocity profile is plug-like for hydrophobic surfaces, and thus viscous dissipation does not occur inside the liquid. In the range of parameters under investigation, the friction coefficient for the $\mathrm{PC}$ surface reaches a maximum at $\sigma_{s} \approx 0.46 \mathrm{C} / \mathrm{m}^{2}$. In contrast, $\lambda$ for the $\mathrm{NC}$ surface increases sharply with $\sigma_{s}$. We do not calculate the friction coefficient for larger surface charge densities because the flow is suppressed significantly and flow inversion occurs as shown in Figure 1b. In addition, at smaller surface charge densities $\sigma_{s}<0.24 \mathrm{C} / \mathrm{m}^{2}$, a very large velocity jump is identified under an applied electric field $E=0.1 \mathrm{~V} / \mathrm{nm}$ (not shown here).
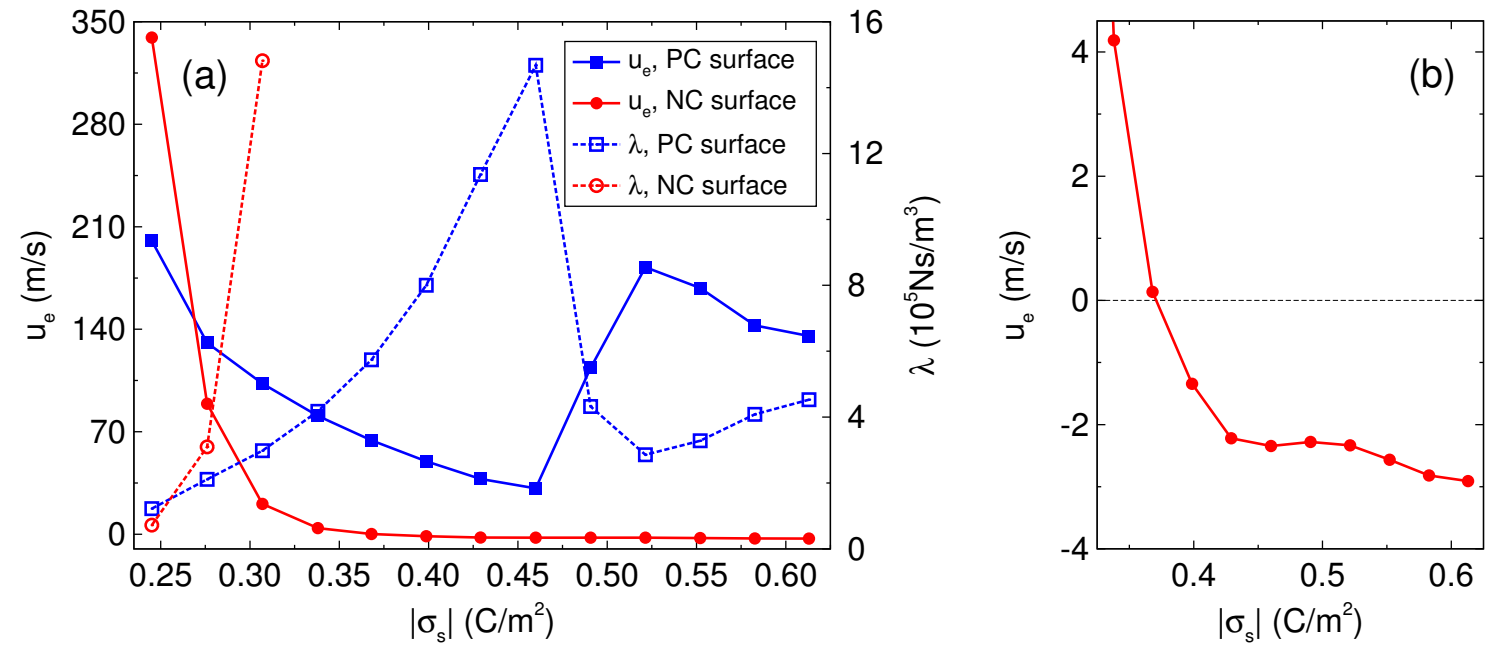

Figure 1. (a) Average electroosmotic flow velocity $u_{e}$ and friction coefficient $\lambda$ as a function of surface charge density $\sigma_{s}$ for positively charged (PC) and negatively charged (NC) surfaces at $E=0.1 \mathrm{~V} / \mathrm{nm}$. (b) shows a zoom-in of $u_{e}$ for the NC surface around the charge reversal.

Clearly, $u_{e}$ grows non-linearly as the electric field increases, as seen in Figure 2. At high surface charge densities, such as $\left|\sigma_{s}\right|=0.613 \mathrm{C} / \mathrm{m}^{2}$, the electroosmotic flow is completely inhibited when the electric field is lower than $0.05 \mathrm{~V} / \mathrm{nm}$. For the NC surface with $\sigma_{s}=-0.613 \mathrm{C} / \mathrm{m}^{2}$, the flow is much weaker compared to the PC surface. This indicates that the surface-fluid interaction becomes stronger at large $\sigma_{s}$ when the surface is negatively charged. However, at $\left|\sigma_{s}\right|=0.184 \mathrm{C} / \mathrm{m}^{2}$, the flow is slightly enhanced for the NC surface. Further, when the surface charge density decreases to 
$\left|\sigma_{s}\right|=0.061 \mathrm{C} / \mathrm{m}^{2}$, the dependence of $u_{e}$ on the surface property and the counterion type is related to the electric field. For the NC surface with the $\mathrm{Na}^{+}$ions as counterions, the electroosmotic mobility $\mu_{e}=u_{e} / E$ is slightly higher at weak electric fields compared to the PC surface (see inset of Figure 2). When the electric field exceeds $0.03 \mathrm{~V} / \mathrm{nm}, \mu_{e}$ for the PC surface with $\sigma_{s}=0.061 \mathrm{C} / \mathrm{m}^{2}$ greatly exceeds the result for the $\mathrm{NC}$ surface with $\sigma_{s}=-0.061 \mathrm{C} / \mathrm{m}^{2}$. This reveals that the electrokinetic transport at charged hydrophobic surfaces is not only dependent on the charge sign of the surface, but also on the magnitude of the surface charge density as well as the electric field. Therefore, it is necessary to explore the effects of the parameters in a larger range for understanding the electrokinetic transport of fluid on charged hydrophobic surfaces.

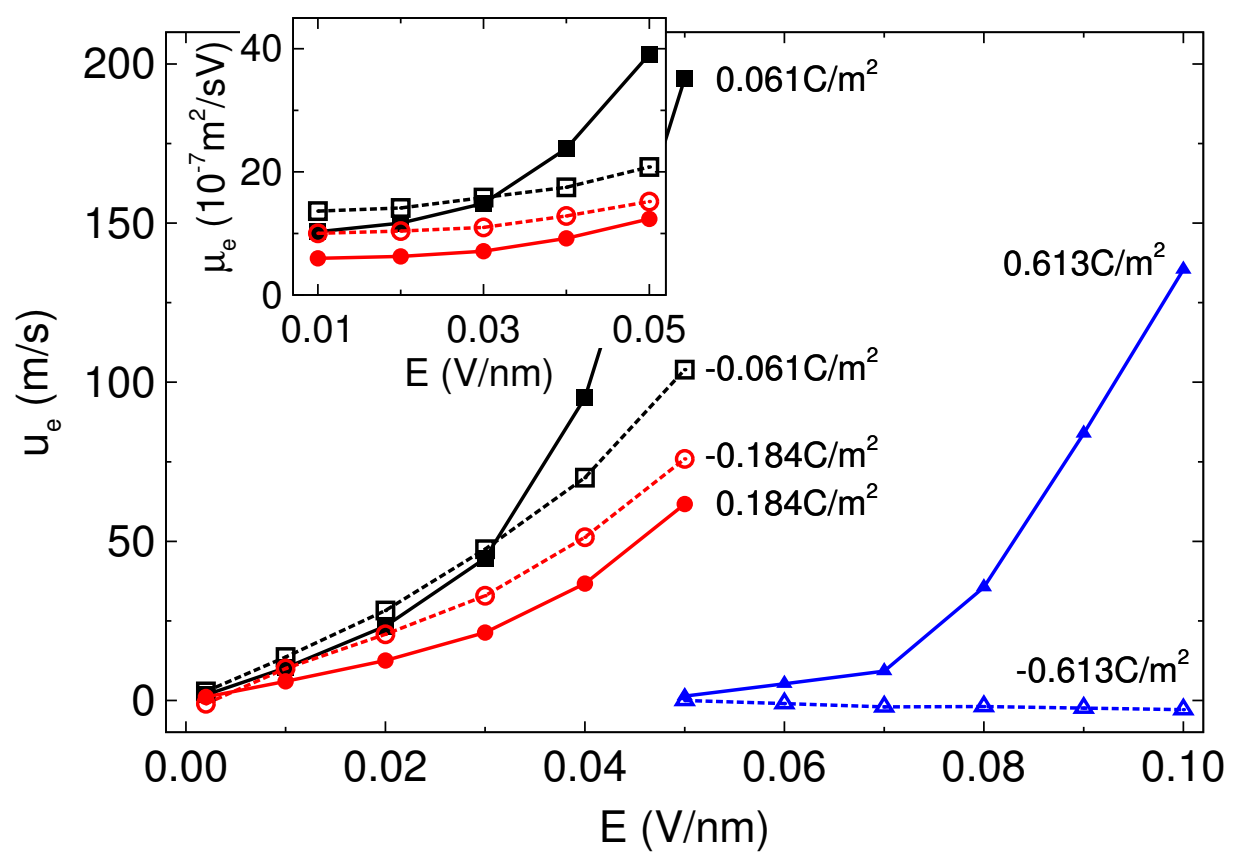

Figure 2. Average electroosmotic flow velocity $u_{e}$ as a function of external electric field $E$ for various surface charge densities. The inset shows the electroosmotic mobility $\mu_{e}=u_{e} / E$ for relatively low surface charge densities.

Figure 3 shows the velocity profiles for different surface charge densities. For the PC surface, the profiles exhibit a perfect plug shape with a velocity jump in the fluid/solid interfacial region, just as the pressure-driven flow in carbon nanotubes [8]. When the surface is negatively charged, we observe that flow inversion occurs at high surface charge density $\sigma_{s}=-0.613 \mathrm{C} / \mathrm{m}^{2}$. This is attributed to the immobilization of adsorbed 
$\mathrm{Na}^{+}$ions due to the strong attraction to the surface, resulting in an effective nonslip boundary. With decreasing surface charge density, the flow inversion disappears. At $\sigma_{s}=-0.429 \mathrm{C} / \mathrm{m}^{2}$, the velocity profile exhibits a pan-like shape. This implies a velocity difference between the $\mathrm{Na}^{+}$and $\mathrm{Cl}^{-}$ion layers. A similar phenomenon was also observed for a NaI solution in hydrophobic nanochannels with low surface charge densities [25].
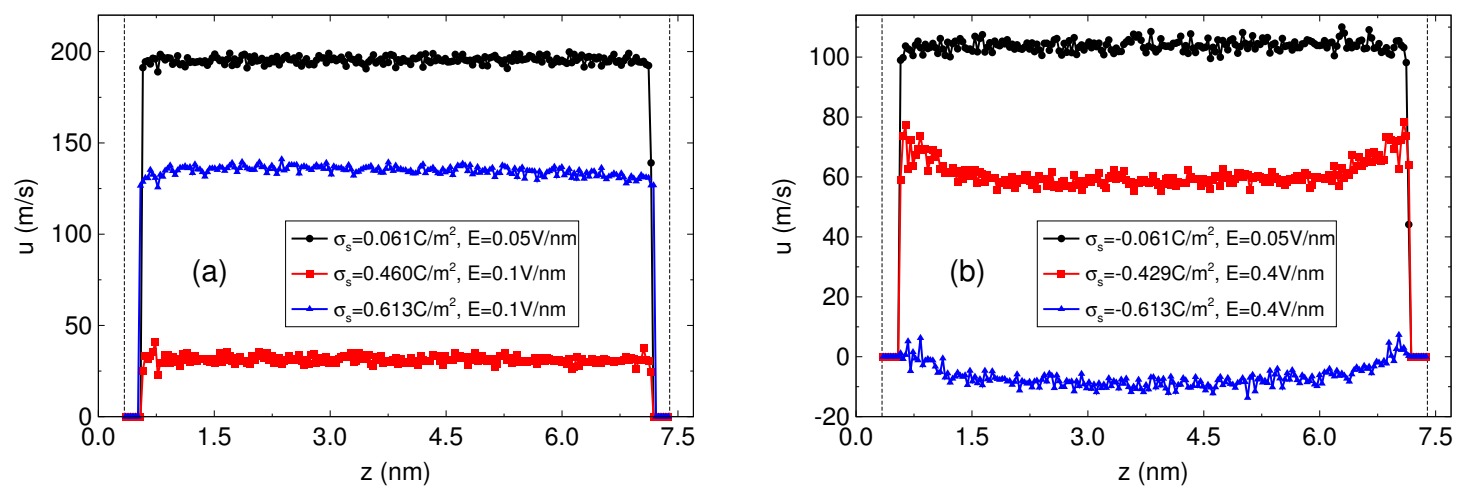

Figure 3. Water velocity profiles across the channel at various surface charge densities and electric fields for the (a) PC and (b) NC surfaces. The vertical dashed lines represent the position of the inner graphene layer. For the PC surfaces, the flow velocity is assumed to be positive along the opposite direction of the $x$ axis.

In Figure 4, we display the ion concentration profiles at various surface charge densities. In the figures, the $\mathrm{Na}^{+}$and $\mathrm{Cl}^{-}$ion concentrations are calculated for the $\mathrm{NC}$ and $\mathrm{PC}$ surfaces, respectively. At $\left|\sigma_{s}\right|=0.061 \mathrm{C} / \mathrm{m}^{2}$ (Figure $4 \mathrm{a}$ ), the first peak of the $\mathrm{Cl}^{-}$ion concentration is higher and closer to the surface compared to the $\mathrm{Na}^{+}$ion concentration. However, the $\mathrm{Na}^{+}$ion concentration shifts towards the surface upon increasing the surface charge density, whereas the position of the first peak of the $\mathrm{Cl}^{-}$ ion concentration stays constant. The ion distributions can help us to explain our puzzling finding that the flow velocity for the $\mathrm{NC}$ surface is higher at relatively low surface charge densities, such as $\left|\sigma_{s}\right| \approx 0.245 \mathrm{C} / \mathrm{m}^{2}$, and the anomalous behavior at larger surface charge densities (see Figure 1). At $\sigma_{s}=-0.245 \mathrm{C} / \mathrm{m}^{2}$ (Figure $4 \mathrm{~b}$ ), only a limited amount of the $\mathrm{Na}^{+}$ions is closer to the surface than the $\mathrm{Cl}^{-}$ions. Totally, the attraction between the $\mathrm{PC}$ surface and the $\mathrm{Cl}^{-}$counterions is still stronger, that is, the friction coefficient is larger. However, when a sufficient amount of $\mathrm{Na}^{+}$ions are 
adsorbed near the surface, such as at $\sigma_{s}=-0.307 \mathrm{C} / \mathrm{m}^{2}$ (Figure $4 \mathrm{c}$ ), the interaction becomes strong enough to reduce the flow velocity to a lower value. At a higher surface charge density $\sigma_{s}=-0.429 \mathrm{C} / \mathrm{m}^{2}$ (Figure $4 \mathrm{~d}$ ), most $\mathrm{Na}^{+}$counterions are strongly bound to the surface. The immobile $\mathrm{Na}^{+}$counterions can not be moved by a low field of $E=0.1 \mathrm{~V} / \mathrm{nm}$.
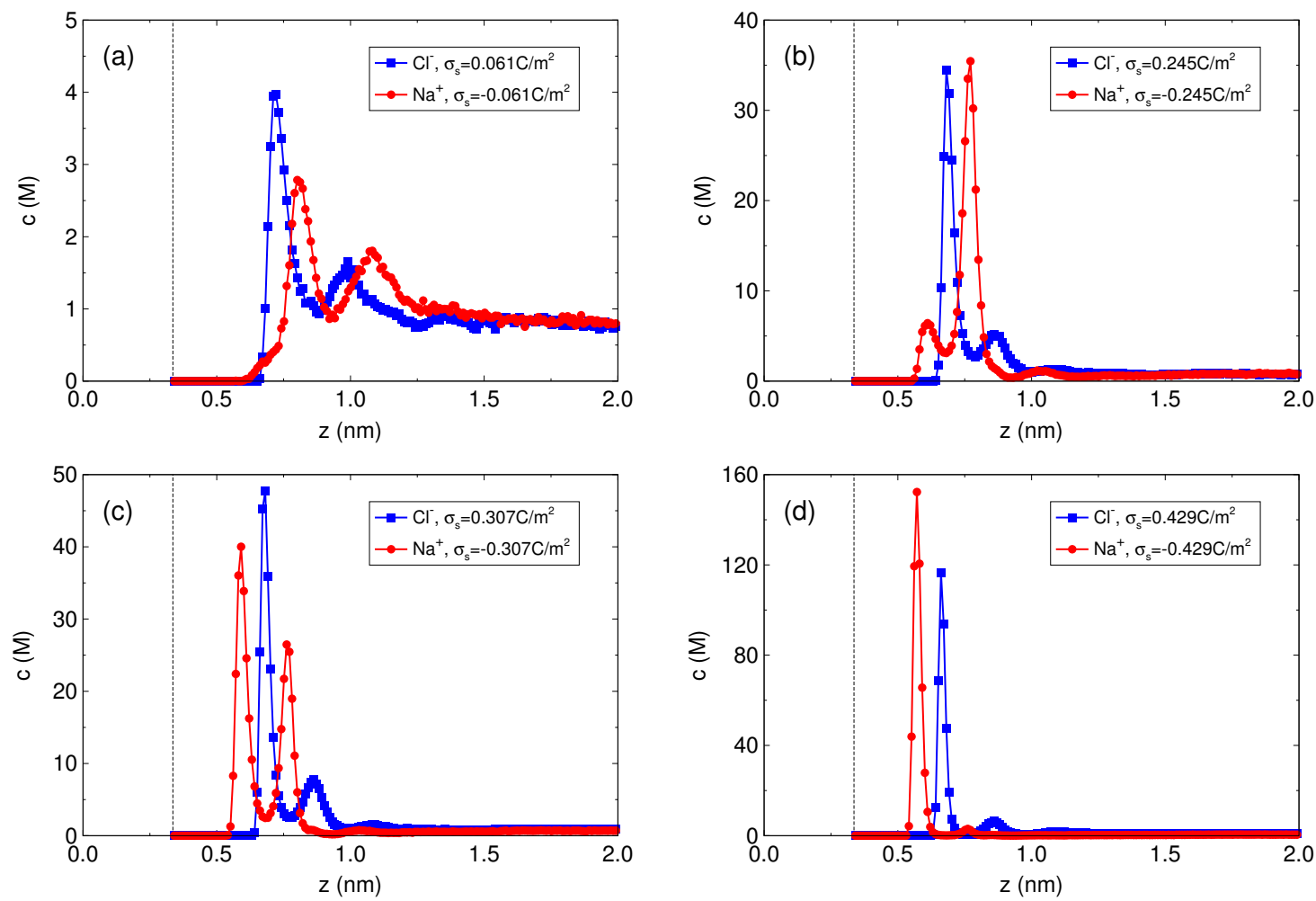

Figure 4. Ion concentrations for various surface charge densities at $E=0.1 \mathrm{~V} / \mathrm{nm}$. $\mathrm{Na}^{+}$and $\mathrm{Cl}^{-}$ion concentrations are shown for negatively and positively charged surfaces, respectively. The vertical dashed line represents the position of inner graphene layer.

Further, we discuss the effects of the surface charge density on the interfacial water structure. Depicted in Figure 5 is the water density $\rho_{w}$ and the average number $N_{h b}$ of hydrogen bonds per water molecule as a function of the distance from the surface. Here, a hydrogen bond is defined such that two water molecules directly interact through a hydrogen bond if the distance between two corresponding oxygen atoms is less than $0.35 \mathrm{~nm}$ and the angle including the hydrogen bond does not exceed $30^{\circ}[8]$. The maximum peak of water density increases with the surface charge density, and shifts towards the surface because of an enhanced electrostatic attraction between the surface 
and the oxygen atoms for the PC surface or the hydrogen atoms for the NC surface. As a result, the interfacial depletion region becomes compressed. In addition, the increase of the height of the first peak also induces a pronounced second peak. Though there is a significant amount of water molecules which cluster near the surface, the hydrogen bonding becomes weaker. Thus, the larger $\sigma_{s}$ undermines the hydrogen bonding ability of water. The fluctuations of water density and ion concentration contribute to oscillations of $N_{h b} . N_{h b}$ approaches the bulk value $(\approx 3.5)$ far away from the surface. At $\left|\sigma_{s}\right|=0.061 \mathrm{C} / \mathrm{m}^{2}, N_{h b}$ in the interfacial region is larger for the $\mathrm{PC}$ surface compared to the NC surface. However, the number of hydrogen bonds for the NC surface becomes larger at higher surface charge densities. This is because the migration of more $\mathrm{Na}^{+}$ions towards the $\mathrm{NC}$ surface triggers relatively strong screening of the surface charges compared to the PC surface. As a result, the effect of the charges at the NC surface on the hydrogen bonding is weakened to some extent. At $\sigma_{s}=0.613 \mathrm{C} / \mathrm{m}^{2}$, the water is completely depleted in the region close to $z=0.7 \mathrm{~nm}$, and thus there are no hydrogen bonds. At $\sigma_{s}=-0.613 \mathrm{C} / \mathrm{m}^{2}$, a slight increase of $N_{h b}$ near the surface is observed compared to the case with $\sigma_{s}=-0.429 \mathrm{C} / \mathrm{m}^{2}$.
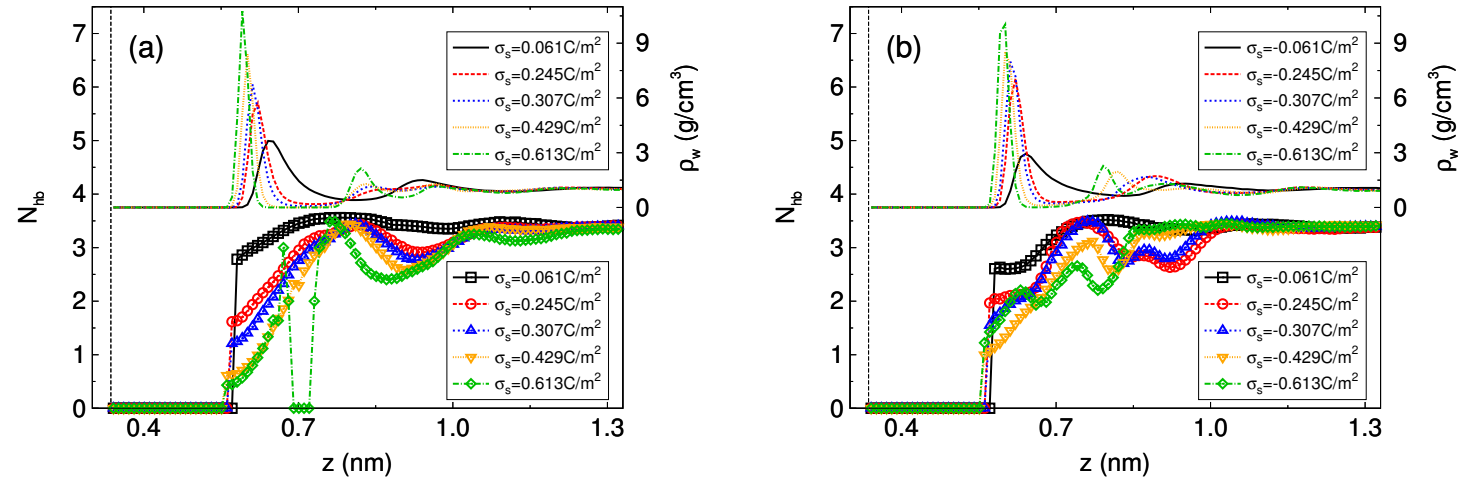

Figure 5. Profiles of the water density (top) and the total number of hydrogen bonds (bottom) at various surface charge densities for the (a) PC and (b) NC surfaces at $E=$ $0.1 \mathrm{~V} / \mathrm{nm}$. The vertical dashed line represents the position of inner graphene layer.

To understand the structure of interfacial water in more details, we also analyze the orientational distribution of the water dipole $P_{\mu}$ and of the $\mathrm{OH}$ bond $P_{O H}$ at various surface charge densities. The distributions as a function of the angle $\theta$ with respect to 
the surface normal are shown in Figure 6. The chosen water molecules are located close to the first peak in the water density. The dipoles of water molecules for the PC surface rotate towards the surface normal as the surface charge density increases (Figure 6a). In particular, at $\sigma_{s}=0.613 \mathrm{C} / \mathrm{m}^{2}$ the strong electrostatic interaction forces the dipoles of most interfacial water molecules to be perpendicular to the surface. When the surface is negatively charged, the dependence of the dipole orientation on the surface charge density becomes more complicated (Figure 6b). When the surface is weakly charged $\left(\sigma_{s}=-0.061 \mathrm{C} / \mathrm{m}^{2}\right)$, the distribution spreads more widely. At $\sigma_{s}=-0.245 \mathrm{C} / \mathrm{m}^{2}$, the dipoles prefer to align with the surface normal. However, the dipoles tilt towards the surface when further increasing the surface charge density. Just as discussed above, due to the migration of $\mathrm{Na}^{+}$counterions to the surface, the preference of the dipole orientation is weakened by the electrostatic screening of $\mathrm{Na}^{+}$counterions. Until the surface carries sufficient charges, such as $\sigma_{s}=-0.613 \mathrm{C} / \mathrm{m}^{2}$, the dipoles again rotate towards the surface normal.

Figure $6 \mathrm{c}$ and d present the distribution of $\mathrm{OH}$ bond orientation. At low surface charge density, most $\mathrm{OH}$ bonds tend to orient parallel to the surface, and water molecules form a planar structure linking each other by hydrogen bonds, corresponding to a large peak at $90^{\circ}$. Such a structure is also found for neutral carbon nanotubes [8]. The increase of the surface charge density induces rotation of $\mathrm{OH}$ bonds which destroys the hydration bonding network. However, the $\mathrm{OH}$ bond distributions are significantly different for the PC and NC surfaces. At $\left|\sigma_{s}\right|=0.061 \mathrm{C} / \mathrm{m}^{2}$, some $\mathrm{OH}$ bonds tend to point towards the bulk fluid for the PC surface (Figure 6 c, i.e. $\theta \approx 20^{\circ}$ ), but towards the wall for the $\mathrm{NC}$ surface (Figure 6 d, i.e. $\theta \approx 160^{\circ}$ ). The increase of the charge density of the PC surface reduces the distribution width. At $\sigma_{s}=0.613 \mathrm{C} / \mathrm{m}^{2}$, the $\mathrm{OH}$ bonds mainly lie in the region between $\theta \approx 40^{\circ}$ and $70^{\circ}$. This reveals that the main population of $\mathrm{OH}$ bonds point towards the fluid. For the NC surface, two pronounced peaks at $\sigma_{s}=-0.613 \mathrm{C} / \mathrm{m}^{2}$ are observed in the range $60^{\circ}<\theta<85^{\circ}$ and $160^{\circ}<\theta<175^{\circ}$, which corresponds to one $\mathrm{OH}$ bond of the water molecule being al- 

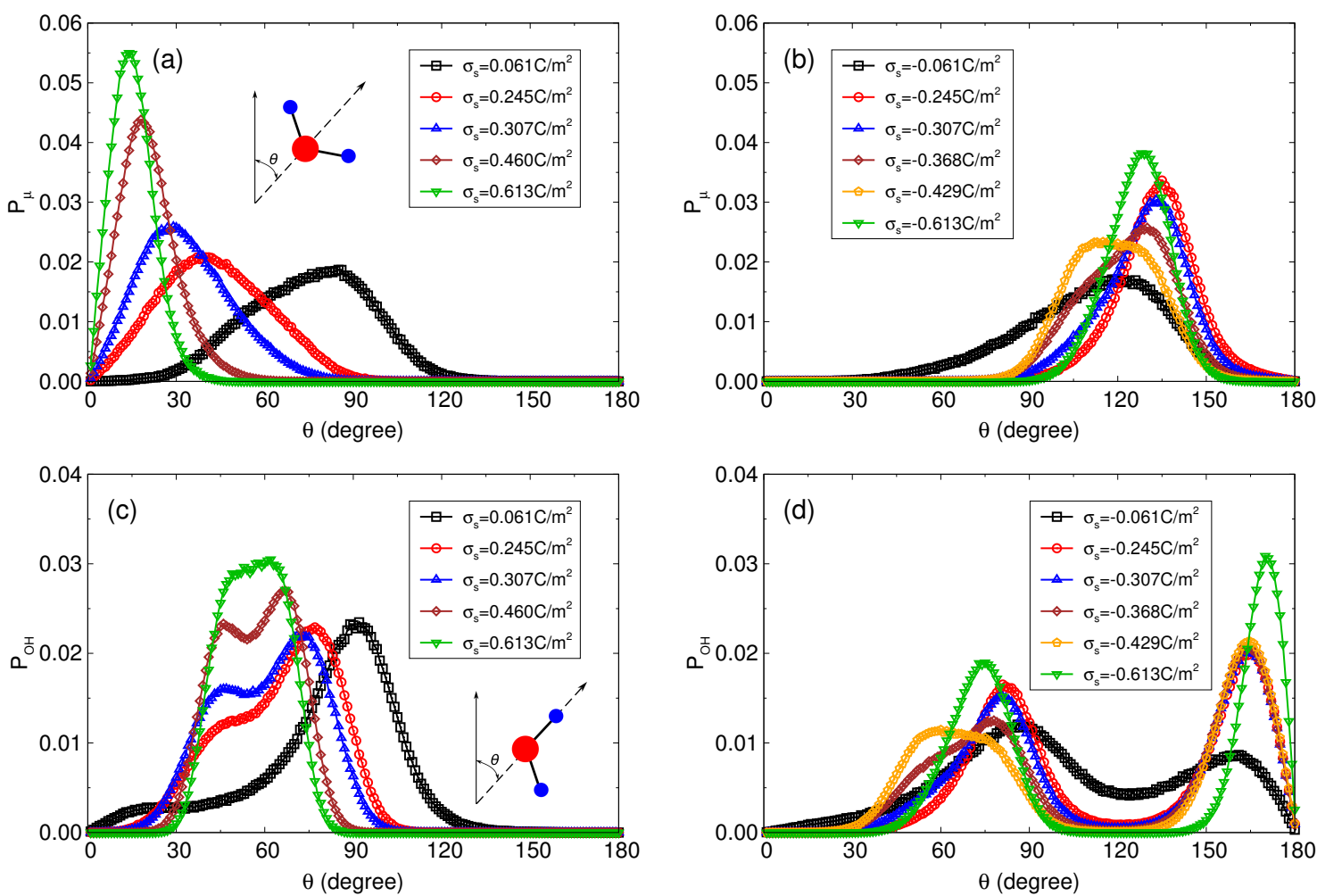

Figure 6. Distributions of (a, b) the interfacial water dipole orientation $P_{\mu}$ and $(\mathrm{c}, \mathrm{d})$ the interfacial $\mathrm{OH}$ bond orientation $P_{O H}$ at the position of the first peak of water density for various surface charge densities. (a, c) and (b, d) correspond to the PC and NC surfaces, respectively.

most perpendicular to the wall. The visual snapshots in Figure 7 give intuitive insight into the interfacial water structure. The water orientation is very sensitive to the properties of the surface. The NC surface (Figure $7 b, d$, and f) attracts positively charged hydrogen atoms leading to one $\mathrm{OH}$ bond of water pointing to the surface, which is consistent with the results in Figure 6d. For the PC surface (Figure 7a, c, and e), the positive surface charges force the $\mathrm{OH}$ bonds to rotate towards the fluid. The increase of surface charge density magnifies these effects. Obviously, a depletion region between the interfacial water layer and the bulk water forms for large surface charge densities $\left|\sigma_{s}\right|=0.429 \mathrm{C} / \mathrm{m}^{2}$ (Figure $7 \mathrm{e}$ and $\mathrm{f}$ ). Moreover, a considerable amount of counterions also cluster in the interfacial region. The $\mathrm{Na}^{+}$ions with a smaller radius than the $\mathrm{Cl}^{-}$ ions are closer to the graphene surface at high surface charge density due to strong electrostatic attraction (Figure 4d).

We further analyze the anomalous increase of $u_{e}$ for the PC surface at high $\sigma_{s} \approx$ 
(a)

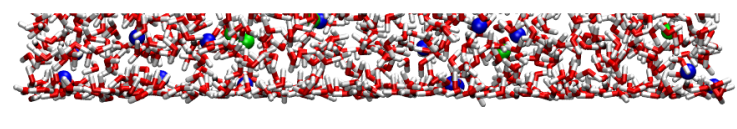

(c)

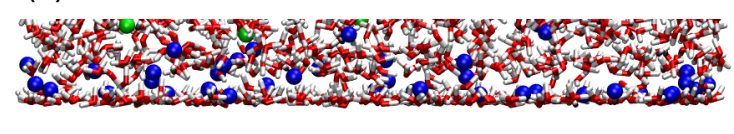

(e)

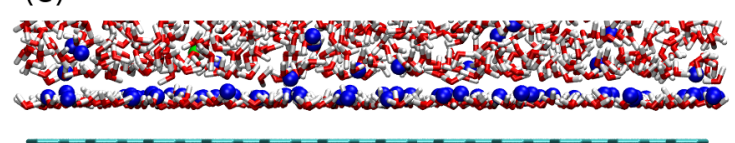

(b)

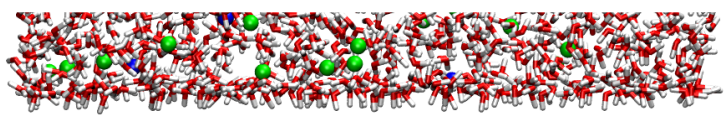

(d)

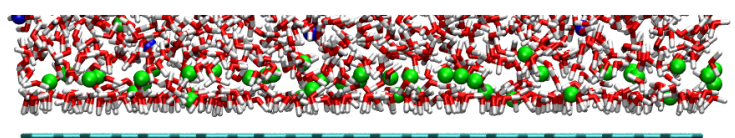

(f)

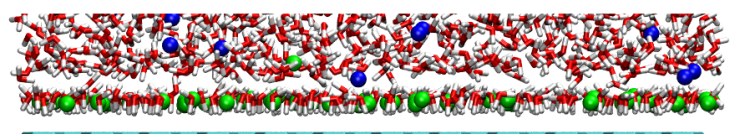

Figure 7. Snapshots of the simulated systems with the (a, c, and e) PC and (b, d, and f) NC surfaces. Only the water molecules close to the surface and the top graphene layer are shown for clarity. The cases from top to bottom correspond to (a and b) $\left|\sigma_{s}\right|=0.061 \mathrm{C} / \mathrm{m}^{2}$, (c and d) $0.245 \mathrm{C} / \mathrm{m}^{2}$ and (e and f) $0.429 \mathrm{C} / \mathrm{m}^{2}$. Color code: water ( $\mathrm{H}$ in white, $\mathrm{O}$ in red), $\mathrm{Na}^{+}$ions (green), $\mathrm{Cl}^{-}$ions (blue), and graphene layer (cyan).

$0.5 \mathrm{C} / \mathrm{m}^{2}$ (see Figure 1a). The enhanced flow means that the friction between the interfacial layer and the surface becomes lower. This behavior seems to be paradoxical because the enhancement of electrostatic interactions with increasing $\sigma_{s}$ should slow down the velocity. To unravel the puzzle at the molecular level, we first present the velocity and concentration of $\mathrm{Cl}^{-}$ions near the surface at $\sigma_{s}=0.46 \mathrm{C} / \mathrm{m}^{2}$ and $0.521 \mathrm{C} / \mathrm{m}^{2}$ in Figure 8 to see if the anomalous flow is caused by the transport of counterions. The $\mathrm{Cl}^{-}$ion velocity is larger at $\sigma_{s}=0.521 \mathrm{C} / \mathrm{m}^{2}$ than at $\sigma_{s}=0.46 \mathrm{C} / \mathrm{m}^{2}$, similar to the
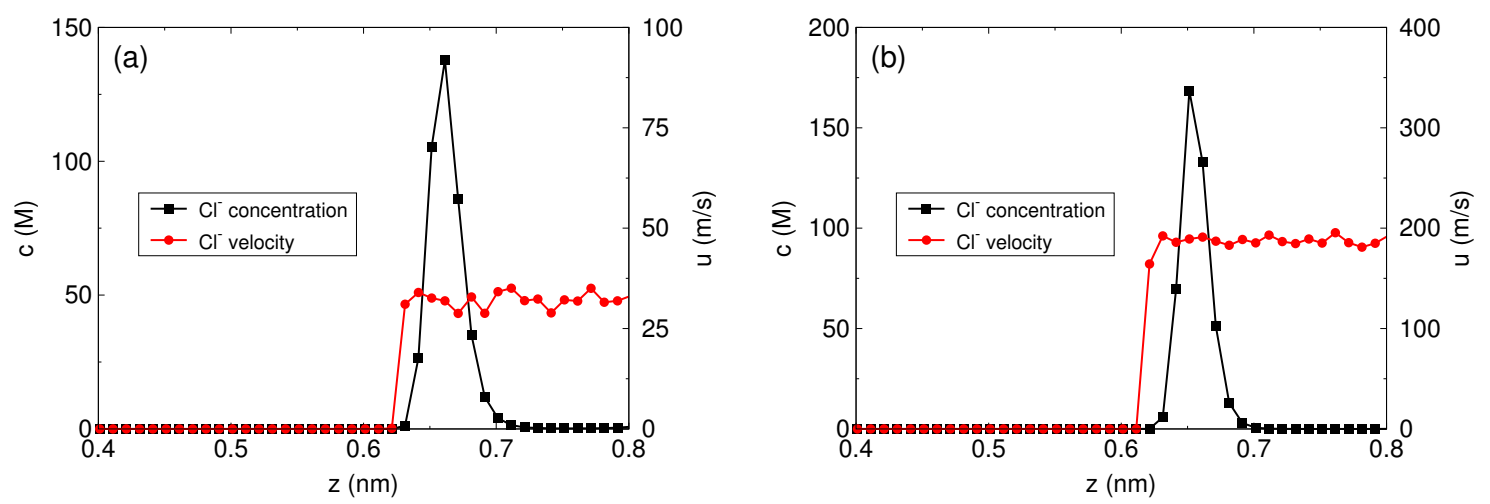

Figure 8. $\mathrm{Cl}^{-}$ion concentration and velocity profiles close to the $\mathrm{PC}$ surfaces at $\sigma_{s}=$ $0.46 \mathrm{C} / \mathrm{m}^{2}$ and $0.521 \mathrm{C} / \mathrm{m}^{2}$. 

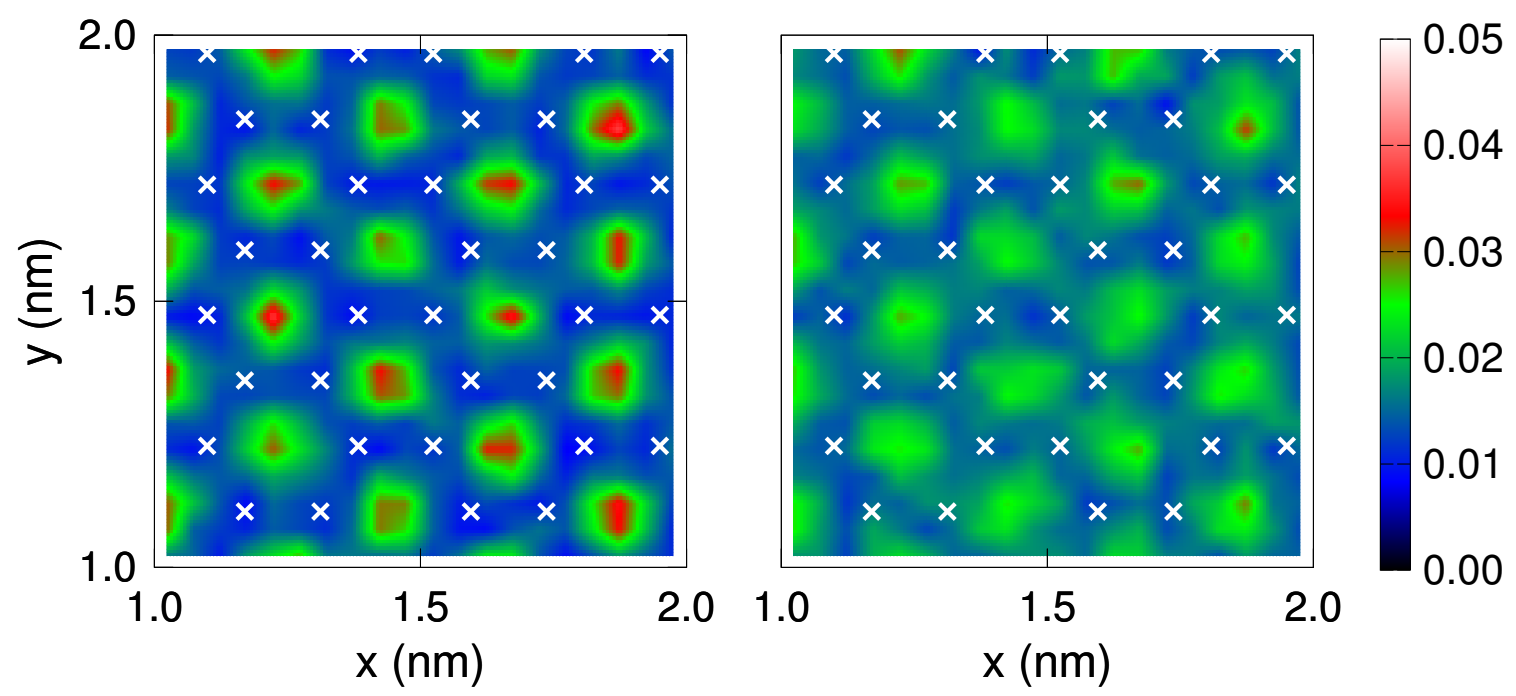

Figure 9. 2D density contours of oxygen atoms for the PC surfaces at (left panel) $\sigma_{s}=$ $0.46 \mathrm{C} / \mathrm{m}^{2}$ and (right panel) $0.521 \mathrm{C} / \mathrm{m}^{2}$ at $E=0.1 \mathrm{~V} / \mathrm{nm}$. Only water molecules belonging to the first layer (distance smaller than $0.36 \mathrm{~nm}$ from the surface) are considered. The cross symbols denote the positions of the graphene atoms.

water flow. No frozen $\mathrm{Cl}^{-}$ions are found in the interfacial region. Further, we show 2D contours of the density of water oxygen atoms in Figure 9 in the first water layer. It is seen that the oxygen atoms exhibit a hexagonal ordering, as observed in simulations for nanotubes with Si atom parameters [8]. This reveals that the water forms a welldefined 2D pattern that correlates with the underlying hexagonal graphene structure. At $\sigma_{s}=0.46 \mathrm{C} / \mathrm{m}^{2}$, the oxygen atoms show a more pronounced ordering in comparison to $\sigma_{s}=0.521 \mathrm{C} / \mathrm{m}^{2}$. In fact, when $\sigma_{s}$ is higher than $0.46 \mathrm{C} / \mathrm{m}^{2}$, the number of water molecules which are trapped is reduced.

We can quantify the motion of the interfacial water by the mean-square displacement (MSD) $\left\langle\left(\mathbf{R}(t)-\mathbf{R}(0)^{2}\right)\right\rangle$. Here, we present the perpendicular component of the MSD in Figure 10 to examine the mobility of the interfacial water due to the influence of the surface charges. In the range of relatively low $\sigma_{s}$, the increase of $\sigma_{s}$ does not affect the diffusion of the interfacial water. However, at higher surface charge densities, such as $\left|\sigma_{s}\right|=0.613 \mathrm{C} / \mathrm{m}^{2}$, the diffusion of water molecules in the interface is significantly reduced. One also notes that the interfacial water shows a very weak mobility for the PC surfaces although the magnitude of the surface charges is equal for the PC and NC surfaces. The weak water diffusion at $\sigma_{s}=0.613 \mathrm{C} / \mathrm{m}^{2}$ is caused by the distribution of 


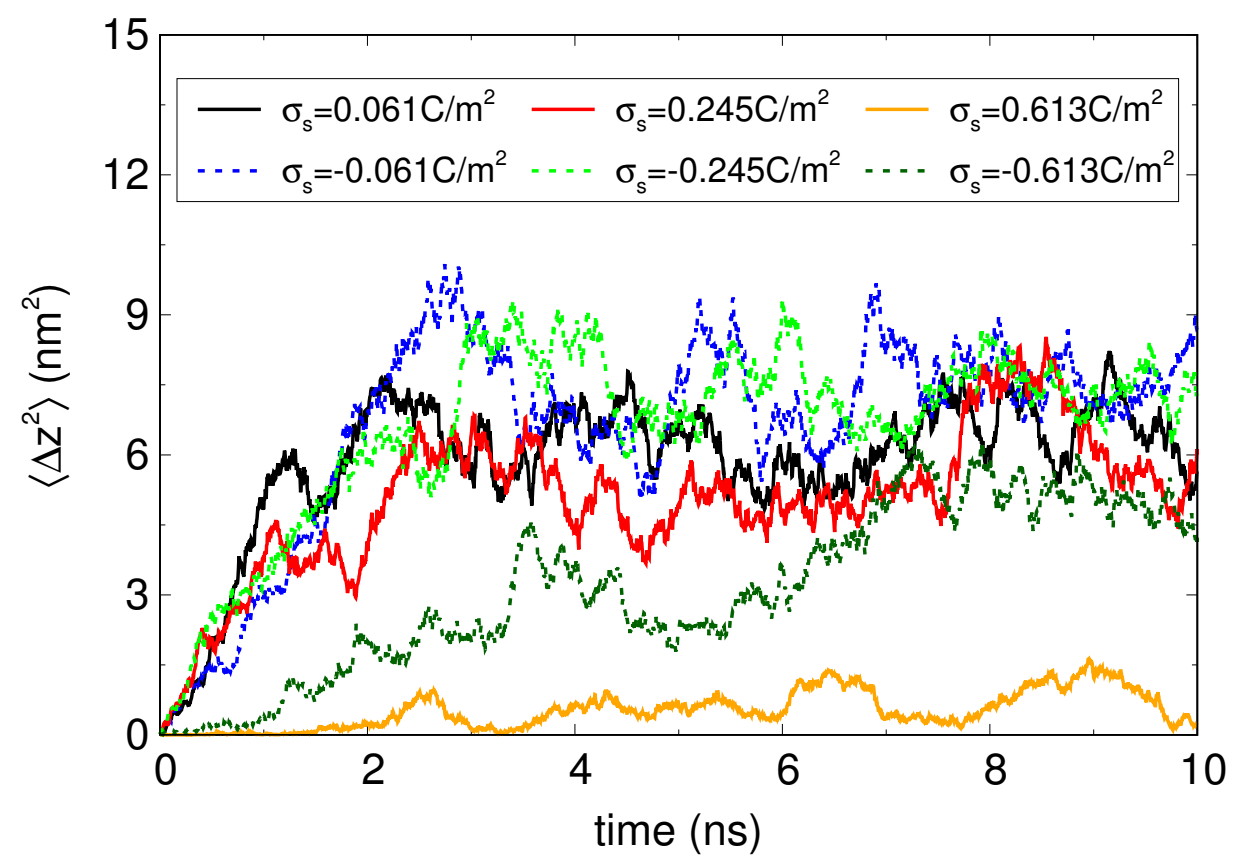

Figure 10. Mean-square displacement of interfacial water diffusion perpendicular to the surface for different surface charge densities at $E=0.1 \mathrm{~V} / \mathrm{nm}$.

the counterions $\left(\mathrm{Cl}^{-}\right.$ions). Compared to the PC surfaces, the counterions $\left(\mathrm{Na}^{+}\right.$ions) of the NC surfaces is closer to the surfaces, which weakens the attraction between the interfacial water and the surface.

Before calculating the electrostatic potential, we first show the interfacial width as a function of the surface charge density based on the Gibbs dividing surface construction (Eq. 4). As shown in Figure 11, $\Delta z_{\text {int }}$ entirely increases with the surface charge density for the PC surface. However, for the NC surface the increase of the surface charge density reduces the Interface width. This is caused by the reorientation of the interfacial water as seen in Figure 7. The electrostatic potential distribution for different surface charge densities is shown in Figure 12. The electrostatic potential is calculated through Eq. 2 with the ionic charge density from the simulation data. We found that for the PC surfaces $\Psi$ decreases rapidly in the depletion region until $z_{\text {int }}$. The interfacial potential increases with the surface charge density. Beyond $z_{\text {int }}$, namely in the bulk region, $\Psi$ decreases with the distance far away from the surface, and then a slower decrease is followed. The point where the slower decrease of $\Psi$ starts is close to the peak of the net charge density. For the NC surfaces, $\Psi$ in the depletion region 


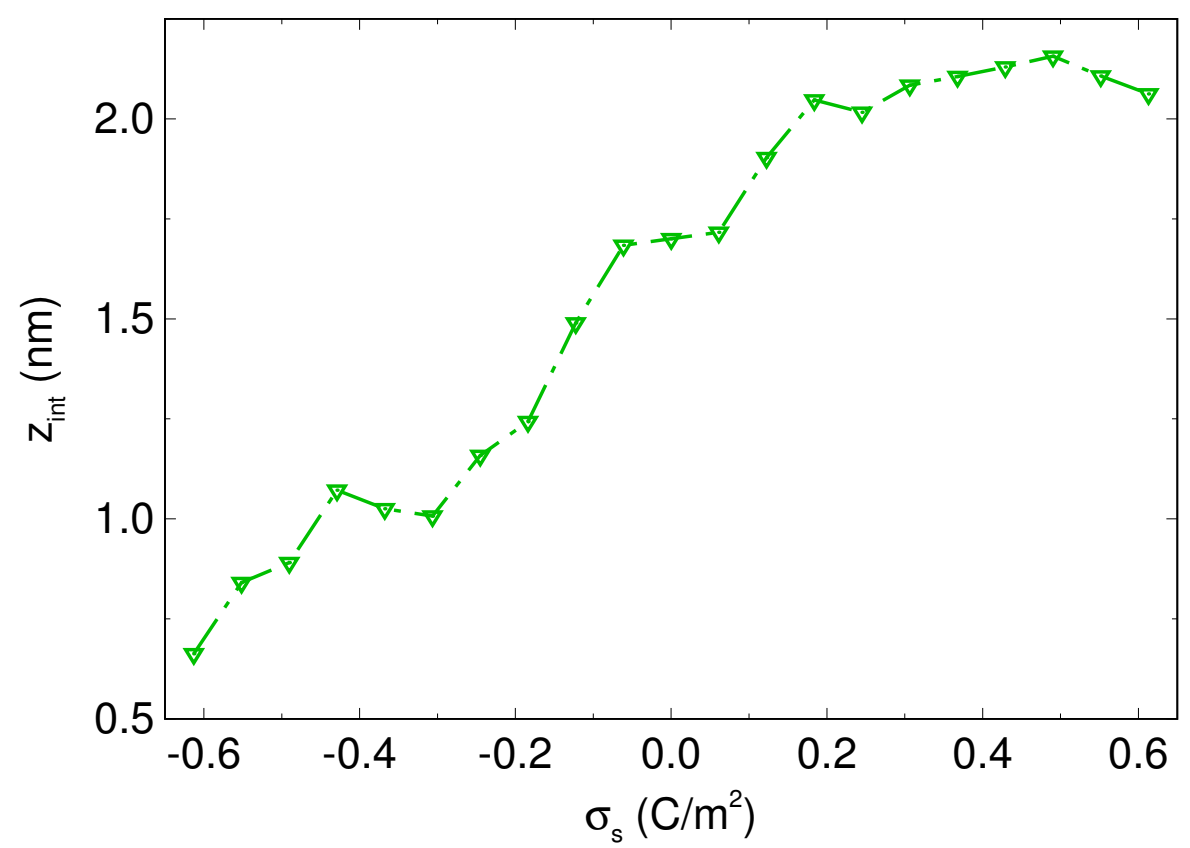

Figure 11. Interfacial layer width $\Delta z_{\text {int }}$ as a function of the surface charge density at $E=0.1 \mathrm{~V} / \mathrm{nm}$. The position of the interface is chosen as the Gibbs dividing surface (Eq. 4).
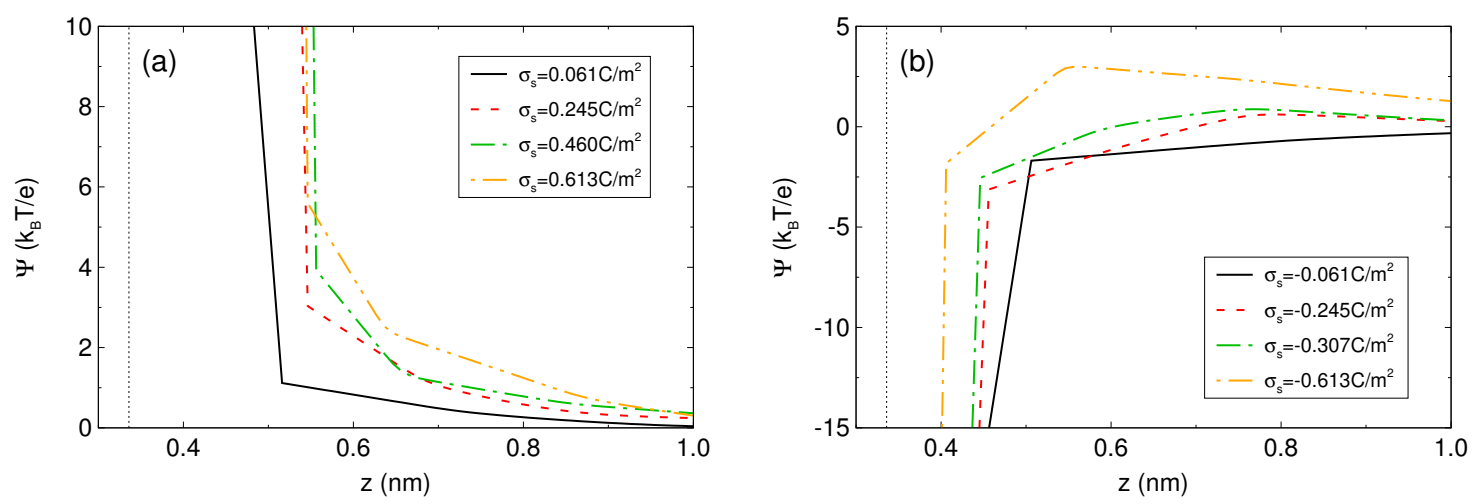

Figure 12. Electrostatic potential $\Psi$ along the direction normal to the surface with different surface charge densities for the (a) PC and (b) NC surfaces. $\Psi$ is calculated using Eq. 2 with the inhomogeneous dielectric profile described in Eq. 3 and the boundary conditions defined in Eq. 5-8. The ionic charge distributions are obtained from the MD simulations.

increases sharply. However, unlike the PC surfaces the interfacial potential shows a more complex dependence on the surface charge density because of the migration of the $\mathrm{Na}^{+}$ions towards the surface owing to the increase of the surface charge density. In the bulk region, $\Psi$ increases with $z$ then followed by a slow decreases until zero.

We present the interfacial potential $\Psi_{\text {int }}$ as a function of the surface charge density in Figure 13. In Figure 13a, the ionic charge distribution is calculated from the 

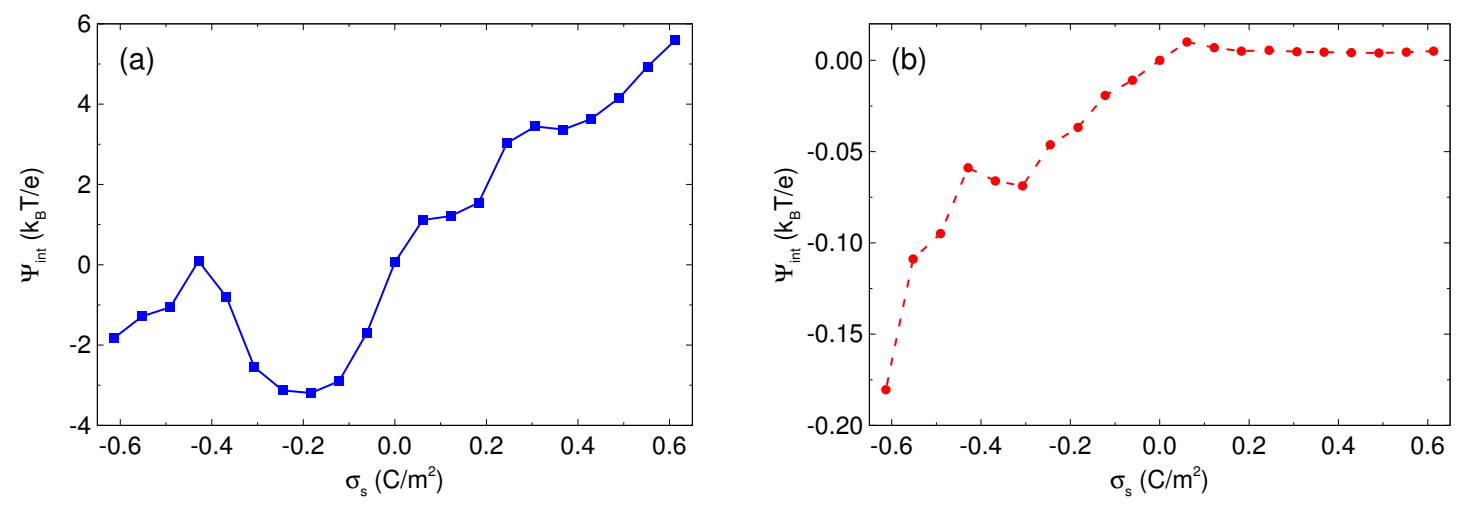

Figure 13. Interfacial electrostatic potential $\Psi_{\text {int }}$ as a function of the surface charge density. In (a), $\Psi_{\text {int }}$ is the interfacial potential which is the electrostatic potential at $z=z_{\text {int }}$ in Figure 12. $z_{i} n t$ is taken as the position $z_{G D S}$ of the Gibbs dividing surface. In (b), $\Psi_{i n t}$ is also the electrostatic potential at $z=z_{\text {int }}$ but is calculated based on Eq. 9 .

simulation data. The interfacial potential for the PC surfaces increases as the surface charge density increases. When the surface is negatively charged, $\Psi_{\text {int }}$ depends nonmonotonically on the surface charge density. At relatively low surface charge densities, $\Psi_{\text {int }}$ shows a minimum at $\sigma_{s} \approx-0.2 \mathrm{C} / \mathrm{m}^{2}$. As the number of the surface charges further increases, the $\mathrm{Na}^{+}$ion distribution shifts towards the surface, leading to an increased $\Psi_{i n t}$. However, when the NC surface has the surface charge density with a higher magnitude than $0.429 \mathrm{C} / \mathrm{m}^{2}$, the interfacial potential decreases again. In Figure 13b, $\Psi_{\text {int }}$ calculated by solving Eq. 9 is rather small. This indicates that the counterion distribution based on the classical PB equation is much closer to the surface because the ion size and the interactions between the particles in the interfacial region are ignored. The change of $\Psi_{\text {int }}$ with the surface charge density also implies ion-specific effects. Note that the classical PB theory predicts a maximum at $\sigma_{s} \approx-0.429 \mathrm{C} / \mathrm{m}^{2}$ (Figure 13b) as the calculation of the interfacial potential using the ionic charge distribution from the MD distribution (Figure 13a). In addition, we also model the system with uncharged graphene surfaces $\left(\sigma_{s}=0\right)$ at $c_{0}=0.71 \mathrm{M}$ to explore the potential of zero charge $(\mathrm{PZC})$. The neutral surface has an interfacial layer width $\Delta z_{\text {int }} \approx 1.7 \AA$ (Figure 11). It was found that no ions are adsorbed to the neutral surfaces except for oscillations of the ion concentrations. The interfacial potential is approximately equal to zero. 
Due to the substantially larger slip length than the characteristic length of the nanochannel, the transport dynamics of the confined fluid is completely determined by the interfacial friction, and not by the viscosity. No direct relationship between the surface potential and the solvent velocity is identified through classical theoretical predictions, such as the Helmholtz-Smoluchowski equation. For the NC surface, the fluid flow is largely suppressed at high surface charge densities, further leading to the flow reversal. In such environments, to explore the effect of the interfacial structure on the fluid dynamics the ionic adsorption needs to be considered. In the present work, though the interfacial potential can not provide direct clue to the solvent velocity, it reveals a complex transition of the interfacial structure caused by the surface charge density for the charged graphene surface.

\section{Conclusions}

To summarize, we have examined the electrokinetic transport of electrolyte at charged graphene surfaces and the water structure in the interface using MD simulations. The effects of surface charge density, ion specificity and electric field strength are explored. We find a strong dependence of the flow velocity on the surface charge. At low surface charge densities, the flow velocity for the NC surface with $\mathrm{Na}^{+}$counterions is larger than that for the $\mathrm{PC}$ surface with $\mathrm{Cl}^{-}$counterions. However, as the surface charge density increases, the electroosmotic flow on the NC surface is significantly suppressed until flow reversal occurs in agreement with previous works [44,48]. The reason for this flow phenomenon is the migration of $\mathrm{Na}^{+}$ions towards the $\mathrm{NC}$ surface at high surface charge densities, which leads to stronger interfacial friction. In contrast, the flow on the PC surface with higher charge densities is enhanced rather than suppressed. Furthermore, an abrupt change in the interfacial water structure induces a transition in the zeta potential. Our analysis gives evidence for the anomalous enhancement of electroosmotic flow at the PC surface. It is found that high surface charge densities decrease the water-surface binding. The reduction of water oxygen binding to the 
surface carbons weakens the friction between the surface and interfacial molecules. The investigation of the diffusion of the interfacial water reveals that the mobility of water molecules in the interfacial region is reduced largely at high surface charge densities. In particular, the interfacial water interacts with the PC surfaces more strongly, which suppresses the water diffusion significantly. Due to the migration of the $\mathrm{Na}^{+}$ions with the increase of the surface charge density, the interfacial potential varies in a more complex manner.

\section{Acknowledgments}

This work was supported by the National Natural Science Foundation of China under Grant No. 31500801, and by the German Science Foundation (DFG) under grant NE810/10. QC gratefully acknowledges support by the Alexander von Humboldt Foundation. We thank Y. Uematsu for helpful discussions.

\section{References}

1 L. Bocquet and E. Charlaix, Chem. Soc. Rev., 2010, 39, 1073-1095.

2 J. C. T. Eijkel and A. van den Berg, Chem. Soc. Rev., 2010, 39, 957-973.

3 D. J. Bonthuis and R. R. Netz, J. Phys. Chem. B, 2013, 117, 11397-11413.

4 L. Bocquet and J.-L. Barrat, Soft Matter, 2007, 3, 685-693.

5 M. Majumder, N. Chopra, R. Andrews and B. J. Hinds, Nature, 2005, 438, 44.

6 J. K. Holt, H. G. Park, Y. Wang, M. Stadermann, A. B. Artyukhin, C. P. Grigoropoulos, A. Noy and O. Bakajin, Science, 2006, 312, 1034-1037.

7 R. R. Netz, Curr. Opin. Colloid Interface Sci., 2004, 9, 192-197.

8 S. Joseph and N. R. Aluru, Nano Lett., 2008, 8, 452-458. 
9 K. Falk, F. Sedlmeier, L. Joly, R. R. Netz and L. Bocquet, Nano Lett., 2010, 10, 4067-4073.

10 K. Falk, F. Sedlmeier, L. Joly, R. R. Netz and L. Bocquet, Langmuir, 2012, 28, $14261-14272$.

11 T. M. Squires and S. R. Quake, Rev. Mod. Phys., 2005, 77, 977-1026.

12 R. B. Schoch, J. Han and P. Renaud, Rev. Mod. Phys., 2008, 80, 839-883.

13 Z. Samec, Electrochim. Acta, 2012, 84, 21-28.

14 N.-S. Choi, Z. Chen, S. A. Freunberger, X. Ji, Y.-K. Sun, K. Amine, G. Yushin, L. F. Nazar, J. Cho and P. G. Bruce, Angew. Chem. Int. Ed., 2012, 51, 9994-10024.

15 P. H. Alijó, F. W. Tavares, E. C. B. Jr. and A. R. Secchi, Electrochim. Acta, 2015, $152,84-92$.

16 A. S. Rathore, Electrophoresis, 2002, 23, 3827-3846.

17 T. Kawamata, M. Yamada, M. Yasuda and M. Seki, Electrophoresis, 2008, 29, $1423-1430$.

18 D. García-Nieto and V. M. Barragán, Electrochim. Acta, 2015, 154, 166-176.

19 C. Davidson and X. Xuan, Electrophoresis, 2008, 29, 1125-1130.

20 D. Gillespie, Nano Lett., 2012, 12, 1410-1416.

21 A. Siria, P. Poncharal, A.-L. Biance, R. Fulcrand, X. Blase, S. T. Purcell and L. Bocquet, Nature, 2013, 494, 455-458.

22 C. Cameselle, Electrochim. Acta, 2015, 181, 31-38.

23 B. Luan and A. Aksimentiev, Phys. Rev. E, 2008, 78, 021912.

24 S. van Dorp, U. F. Keyser, N. H. Dekker, C. Dekker and S. G. Lemay, Nat. Phys., 2009, 5, 347-351. 
25 D. M. Huang, C. Cottin-Bizonne, C. Ybert and L. Bocquet, Phys. Rev. Lett., 2007, 98, 177801.

26 F. H. J. van der Heyden, D. Stein, K. Besteman, S. G. Lemay and C. Dekker, Phys. Rev. Lett., 2006, 96, 224502.

27 R. Qiao and N. R. Aluru, Nano Lett., 2003, 3, 1013-1017.

28 Q. Cao, Microfluid. Nanofluid., 2016, 20, 132.

29 L. Joly, C. Ybert, E. Trizac and L. Bocquet, Phys. Rev. Lett., 2004, 93, 257805.

30 D. M. Huang, C. Cottin-Bizonne, C. Ybert and L. Bocquet, Phys. Rev. Lett., 2008, 101, 064503.

31 D. M. Huang, C. Cottin-Bizonne, C. Ybert and L. Bocquet, Langmuir, 2008, 24, $1442-1450$.

32 S. Chakraborty, Phys. Rev. Lett., 2008, 100, 097801.

33 D. Kim and E. Darve, J. Colloid Interface Sci., 2009, 330, 194-200.

34 A. V. Belyaev and O. I. Vinogradova, Phys. Rev. Lett., 2011, 107, 098301.

35 J. Chakraborty, S. Pati, S. K. Som and S. Chakraborty, Phys. Rev. E, 2012, 85, 046305 .

36 S. Chakraborty, D. Chatterjee and C. Bakli, Phys. Rev. Lett., 2013, 110, 184503.

37 D. J. Bonthuis and R. R. Netz, Langmuir, 2012, 28, 16049-16059.

38 S. R. Maduar, A. V. Belyaev, V. Lobaskin and O. I. Vinogradova, Phys. Rev. Lett., $2015, \mathbf{1 1 4}, 118301$.

39 C. I. Bouzigues, P. Tabeling and L. Bocquet, Phys. Rev. Lett., 2008, 101, 114503.

40 D. Ben-Yaakov, D. Andelman, D. Harries and R. Podgornik, J. Phys.: Condens. Matter, 2009, 21, 424106. 
41 I. Borukhov, D. Andelman and H. Orland, Phys. Rev. Lett., 1997, 79, 435-438.

42 G. Tresset, Phys. Rev. E, 2008, 78, 061506.

43 A. R. J. Silalahi, A. H. Boschitsch, R. C. Harris and M. O. Fenley, J. Chem. Theory Comput., 2010, 6, 3631-3639.

44 R. Qiao and N. R. Aluru, Phys. Rev. Lett., 2004, 92, 198301.

45 M. Rezaei, A. R. Azimian and D. T. Semiromi, Heat Mass Transfer., 2015, 51, 661670.

46 S. Prakash, H. A. Zambrano, M. Fuest, C. Boone, E. Rosenthal-Kim, N. Vasquez and A. T. Conlisk, Microfluid. Nanofluid., 2015, 19, 1455-1464.

47 R. Hartkamp, B. Siboulet, J.-F. Dufrêche and B. Coasne, Phys. Chem. Chem. Phys., $2015, \mathbf{1 7}, 24683-24695$.

48 H. Yoshida, H. Mizuno, T. Kinjo, H. Washizu and J.-L. Barrat, J. Chem. Phys., $2014, \mathbf{1 4 0}, 214701$.

49 M. D. Ellison, S. Menges, L. Nebel, G. DArcangelo, A. Kramer, L. Drahushuk, J. Benck, S. Shimizu and M. S. Strano, J. Phys. Chem. C, 2017, 121, 2005-2013.

50 H. Wang, J. Varghese and L. Pilon, Electrochim. Acta, 2011, 56, 6189-6197.

51 X. Liu, Y. Wang, S. Li and T. Yan, Electrochim. Acta, 2015, 184, 164-170.

52 J. Wang, P. Cieplak and P. A. Kollman, J. Comput. Chem., 2000, 21, 1049-1074.

53 D. E. Smith and L. X. Dang, J. Chem. Phys., 1994, 100, 3757-3766.

54 T. Werder, J. H. Walther, R. L. Jaffe, T. Halicioglu and P. Koumoutsakos, J. Phys. Chem. B, 2003, 107, 1345-1352.

55 H. J. C. Berendsen, J. R. Grigera and T. P. Straatsma, J. Phys. Chem., 1987, 91, $6269-6271$. 
56 J.-P. Ryckaert, G. Ciccotti and H. J. Berendsen, J. Comput. Phys., 1977, 23, 327341.

57 R. W. Hockney and J. W. Eastwood, Computer Simulation Using Particles, Adam Hilger, Bristol, 1988.

58 I.-C. Yeh and M. L. Berkowitz, J. Chem. Phys., 1999, 111, 3155-3162.

59 S. Plimpton, J. Comput. Phys., 1995, 117, 1-19.

60 S. Nosé, J. Chem. Phys., 1984, 81, 511-519.

61 W. G. Hoover, Phys. Rev. A, 1985, 31, 1695-1697.

62 Y. Uematsu, R. R. Netz and D. J. Bonthuis, Chem. Phys. Lett., 2017, 670, 11-15.

63 L. Joly, C. Ybert, E. Trizac and L. Bocquet, J. Chem. Phys., 2006, 125, 204716. 